\title{
ANALYSIS AND RECOGNITION OF PAIN IN 2D FACE IMAGES OF FULL TERM AND HEALTHY NEWBORNS
}

\author{
Gilberto F. Teruel ${ }^{1}$, Tatiany M. Heiderich ${ }^{2}$, Ruth Guinsburg $^{2}$, Carlos E. Thomaz $^{1}$ \\ ${ }^{1}$ Centro Universitário FEI \\ São Bernardo do Campo, SP, Brazil \\ ${ }^{2}$ Universidade Federal de São Paulo (UNIFESP) \\ São Paulo, SP, Brazil \\ \{gilberto.ferreira.teruel, tatianyms\} @gmail.com.br \\ ruthgbranetpoint.com.br, cet@fei.edu.br
}

\begin{abstract}
This paper proposes a sequence of computational procedures for detecting, interpreting and classifying patterns in frontal two-dimensional images of faces for automatic recognition of pain in newborns. Using data transformation and extraction of statistical characteristics from a real-life, healthy-term newborn image database, it was possible to interpret and model the subjectivity of trained health professionals, quantifying human knowledge in the task of recognizing pain enabling automatic identification. These results were compared with NFCS based classifications by the same professionals of the same images.
\end{abstract}

Resumo. Esse artigo propõe uma sequência de procedimentos computacionais para detecção, interpretação e classificação de padrões em imagens bidimensionais frontais de faces para reconhecimento automático de dor em recémnascidos. Usando transformações de dados e extrações de características estatísticas de um banco de imagens reais de recém-nascidos a termo e saudáveis, foi possivel abstrair e modelar a subjetividade dos profissionais de saúde treinados, quantificando o conhecimento humano na tarefa de reconhecimento de dor permitindo uma identificação automática. Tais resultados foram comparados com classificações das mesmas imagens, com base na escala de dor NFCS, pelos mesmos profissionais.

\section{INTRODUÇÃO}

A percepção da dor é intrínseca a qualquer ser vivo que possui sistema nervoso central [Anand and Craig 1996]. Nos seres humanos, essa experiencia sensorial desencadeia reações fisiológicas incômodas e emocionalmente ruins. No entanto, a dor é essencial para a sobrevivência, pois indica presença de dano corporal ou ameaça de morte, promovendo um comportamento de fuga [Luda et al. 2007].

Para seres humanos adultos, a dor pode ser identificada por meio de expressões verbais e indicações subjetivas. Porém, tal identificação não é possível quando o indivíduo está inconsciente ou impossibilitado de se comunicar [Guinsburg and Cuenca 2010]. Em lactentes pré-verbais existe essa mesma dificuldade em identificar a dor e intensidade que a mesma se manifesta, devido à impossibilidade de comunicação verbal. Sendo 
assim, a identificação da dor se dá por meio da análise de ações e reações, não necessariamente objetivas, do recém-nascido frente ao estímulo desagradável ou doloroso [Guinsburg and Cuenca 2010].

As consequências dessa experiência dolorosa em recém-nascidos são mais severas dado a imaturidade neurológica e exposição a diversos procedimentos em unidades de terapia intensiva neonatal, assim como o uso irregular de analgesia e sedação. Essas consequências tornam os recém-nascidos mais sensíveis e susceptíveis a experiências estressantes e potencialmente dolorosas, que podem propiciar impacto negativo na evolução neurológica, cognitiva e psiquiátrica deste indivíduo a curto e médio prazos [Grunau 2013, Guinsburg 1999, Chermont et al. 2003, Heiderich et al. 2015, Aymar and Coutinho. 2008]. Portanto, diversas escalas de dor não-invasivas tem sido desenvolvidas, baseadas em observações de comportamento, como movimentos faciais, podendo ou não serem mescladas com indicadores fisiológicos [Lawrence et al. 1993, Stevens et al. 1996, Grunau and Craig 1987, Krechel and Bildner 1995]. Essas escalas e métodos de avaliação permitem a investigação e criação de métodos de avaliação nãoinvasivos e mais específicos do fenômeno doloroso, que possibilitam o supervisionamento automático do recém-nascido em um período de tempo maior [Heiderich et al. 2015], auxiliando não somente a identificação mais precoce, como também uma compreensão melhor desta experiência negativa.

Nesse contexto de escalas de dor e tomando-se como referência o trabalho pioneiro sobre dor neonatal avaliada por meio de software que detecta movimentos faciais de [Heiderich et al. 2015], o presente artigo fundamenta-se na hipótese de que imagens frontais de faces podem ser processadas computacionalmente e características baseadas nas mímicas faciais possam ser extraídas, identificando a existência de dor e sua correlação com níveis de intensidade de dor auxiliando no diagnóstico em recém-nascidos.

O arcabouço computacional proposto e implementado aqui assemelha-se ao processo de avaliação subjetivo aplicado por seres humanos, quando baseado em técnicas de análise de dor, estimulando a aplicação de métodos computacionais de detecção e segmentação de objetos, normalização espacial de imagens de faces, algoritmos de extração de características e métodos estatísticos para treinamento de profissionais da saúde e possivelmente o uso a beira de leito para auxílio na identificação de dor em recém-nascidos.

\section{MATERIAIS E MÉTODOS}

\subsection{Banco de imagens}

O banco de imagens de recém-nascidos contém imagens capturadas durante procedimentos potencialmente dolorosos e/ou estressantes necessários aplicado aos recém-nascidos [Heiderich et al. 2015]. Esse banco é composto por 360 amostras de imagens de 30 recém-nascidos diferentes, com idade gestacional entre 34 e 41 semanas e entre 24 e 168 horas de vida (prematuros tardios ou a termo), alimentados e saudáveis (sem más formações congênitas, anomalias faciais, necessidade de suporte ventilatório, sonda gástrica ou injeções intramusculares e/ou subcutâneas) [Heiderich et al. 2015].

As imagens foram dispostas de maneira aleatória e avaliadas por profissionais da área da saúde com especialização em neonatologia, de forma subjetiva e também com base na escala de dor NFCS - Neonatal Facial Coding System [], classificando a imagem como "Sem Dor"ou "Com Dor". 


\subsection{Metodologia}

A metodologia proposta pode ser vista na Figura 1 e é composta das seguintes etapas principais: pré-processamento, contemplando segmentação de faces e redimensionamento; marcação dos pontos fiduciais; normalização espacial das imagens, utilizando transformações não-lineares (AFIM e FFD); construção do Atlas de Referência Inicial e Atlas de Recém-Nascidos e análise estatística multivariada, usando PCA (Principal Component Analysis), para encontrar as componentes principais cujos dados apresentam maior variância em relação aos conjuntos "Com Dor"e "Sem Dor", além de reduzir a dimensionalidade da informação, e LDA (Linear Discriminant Analysis), para encontrar a característica mais discriminante e classificar as imagens em escala contínua entre "Sem Dor"e "Com Dor".

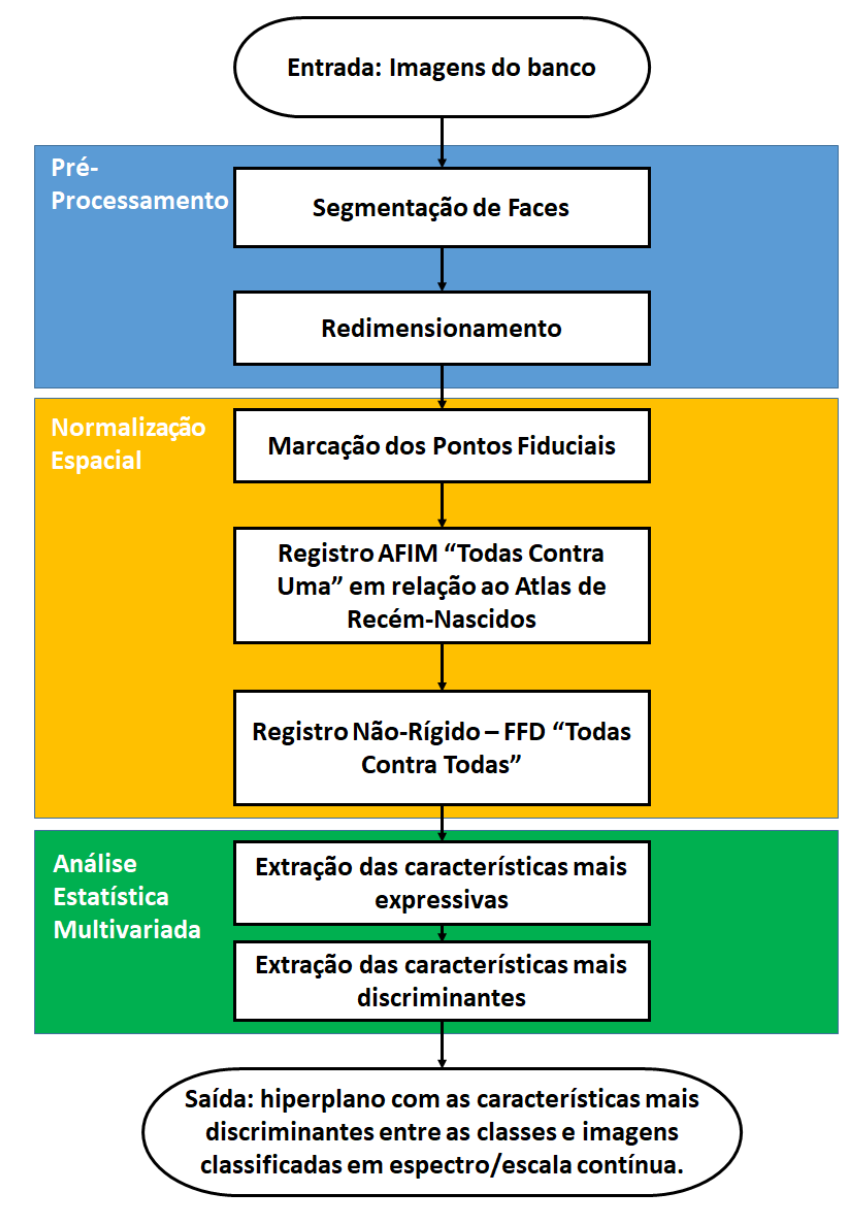

Figura 1. Fluxograma da metodologia proposta e aplicada nesse estudo.

\subsubsection{Pré-Processamento}

Para analisar e comparar as características entre imagens, as mesmas devem estar espacialmente alinhadas a um sistema de coordenadas comum possibilitando a extração das regiões de interesse da imagem, nesse caso a face do recém-nascido [Amaral et al. 2009, Xavier et al. 2016]. 
Para extrair a região da imagem que contém a face a ser estudada é necessário identificar os pixels de interesse. Através da técnica HOG - histograma de gradientes orientados, uma janela deslizante percorre a imagem gerando vetores de intensidade com características do formato do objeto, em conjunto com um classificador linear treinado (SVM-Support Vector Machine) define-se a região a ser extraída. Esses métodos em conjunto permitem a identificação dos locais das faces, mesmo em imagens com ruído, artefatos e baixa/alta luminância [Dalal and Triggs 2005].

\subsubsection{Marcação dos Pontos Fiduciais}

Para que seja possível normalizar espacialmente as imagens, se faz necessário encontrar os pontos de controle que descrevem a face do recém-nascido e preservam as características a serem estudadas [Rueckert et al. 1999].

Nessa metodologia utiliza-se um algoritmo baseado em regressores em cascata, publicado em [Kazemi and Sullivan 2014], o qual permite estimar pontos fiduciais em faces de seres humanos presentes em imagens bidimensionais. Os regressores em cascata atuam de forma a contribuir para o deslocamento correto desses pontos fiduciais estimados em direção a posição esperada, gerando uma marcação automática de 68 pontos que descrevem o rosto do recém-nascido da imagem, como pode ser visto genericamente na Figura 2.

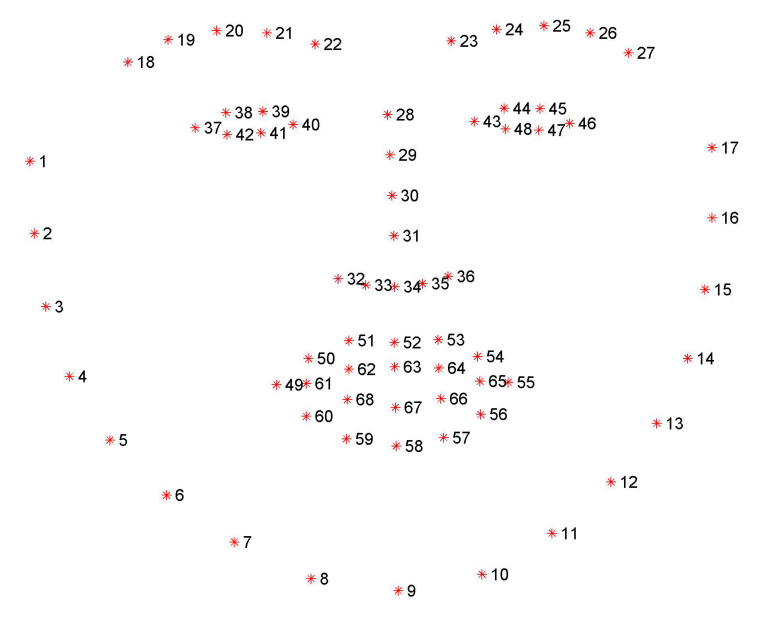

Figura 2. Representação dos 68 pontos fiduciais marcados sobre face humana genérica. Imagem adaptada de [Sagonas et al. 2013]

\subsubsection{Normalização Espacial das Imagens}

O objetivo dessa etapa é alinhar espacialmente as características a serem comparadas e analisadas. Esse alinhamento é feito através de transformações rígidas e não-rígidas, aplicadas a cada imagem do banco de recém-nascidos em relação a uma imagem de referência. Na prática, essa transformação reduz as diferenças devidas a orientação espacial, escala, posição e diferenças nas formas das faces [Amaral et al. 2009, Rueckert et al. 1999]. 
Nessa metodologia utiliza-se a transformação AFIM, generalização da transformação rígida, com o objetivo de transladar, rotacionar e alterar a escala, alinhando o eixo geométrico da face do recém-nascido na imagem com a face da imagem referência, sem causar deformações. A fim de realizar um alinhamento ótimo é utilizado também a transformação não-rígida FFD-Free Form Deformation permitindo uma transformação local, de maneira que os pixels das regiões próximas aos pontos fiduciais sejam deslocados e tendam aos pontos de referência da face na imagem objetivo.

\subsubsection{Extração das Características Mais Expressivas}

Com as faces dos recém-nascidos das imagens do banco alinhadas pode ser realizado as análises multivariadas estatísticas com o objetivo realizar análises conjuntas e simultâneas de duas ou mais variáveis correlacionadas entre si.

Aqui as variáveis a serem analisadas e correlacionadas são os pixels das faces nas imagens do banco, para encontrar as características que mais variam ou discriminam o conjunto de imagens utiliza-se a técnica PCA - Análise de Componentes Principais [Fukunaga 1990]. Técnica geralmente utilizada para reduzir a dimensionalidade de um conjuntos de variáveis correlacionadas entre si, através de transformação linear. O novo conjunto de dados apresenta uma quantidade menor ou igual de variáveis em relação aos dados originais, sendo que essas novas variáveis passam a estar em um novo sistema de coordenadas, onde cada eixo é ortogonal entre si. Para que haja redução de dimensionalidade, componentes de menor variância são descartadas, trazendo uma perda de informação controlada, mantendo as características mais relevantes [Fukunaga 1990].

Expressão genérica que define o hiperplano poder ser vista na seguinte equação

$$
\boldsymbol{y}=\overline{\boldsymbol{x}}+j \cdot \sqrt{\lambda_{i}} \cdot \boldsymbol{p}_{i},
$$

onde $\overline{\boldsymbol{x}}$ é a média global do conjunto; $j \in\{-3,-2,-1,0,1,2,3\} ; \Lambda_{i}$ é o autovalor cujo $p_{i}$ é o autovetor correspondente.

Nesse estudo o PCA foi utilizado a fim de analisar as características mais expressivas com maiores variâncias, sendo possível identificar as expressões faciais mais relevantes em termos de variância intra classes "Sem Dor"e "Com dor". Foi possível também reduzir a dimensionalidade dos dados e gerar imagens sintéticas com as principais características de cada classe a partir do hiperplano criado, contribuindo para uma extensão do banco de imagens original.

\subsubsection{Características Mais Discriminantes}

A técnica PCA não garante que todas as características faciais estejam presentes em apenas uma componente principal, possibilitando apenas a distinção das classes de interesse. Para encontrar um hiperplano onde todas as características a serem analisadas estejam presentes e se alterem em conjunto e de forma contínua de uma classe para outra é necessário utilizar técnicas supervisionadas de análise multivariada estatística como o LDA Análise Discriminante Linear. 
O LDA é uma generalização da proposta original desenvolvida por Ronald A. Fisher [Fisher 1936]. Técnica estatística utilizada com o propósito de separar os conjuntos "Sem Dor"e "Com Dor", através de combinações lineares das variáveis que caracterizam cada classe, com o objetivo de maximizar a distância entre classes e minimizar a dispersão (variabilidade) de dados dentro de cada classe [Thomaz et al. 2006]. Para isso o critério de Fisher deve ser satisfeito, encontrando uma matriz de projeção $\boldsymbol{P}_{l d a}$ que maximize a razão entre o determinante da matriz de espalhamento inter-classes $\boldsymbol{S}_{b}$ e o determinante da matriz de espalhamento intra-classes $\boldsymbol{S}_{w}$, formulado na equação

$$
\boldsymbol{P}_{l d a}=\underset{P}{\operatorname{argmax}} \frac{\left|\boldsymbol{P}^{T} \boldsymbol{S}_{b} \boldsymbol{P}\right|}{\left|\boldsymbol{P}^{T} \boldsymbol{S}_{w} \boldsymbol{P}\right|}
$$

A Equação (2) do critério de Fisher é satisfeita quando o argumento $\boldsymbol{P}_{l d a}$ é composto de $g-1$ autovetores de $\boldsymbol{S}_{w}^{-1} \boldsymbol{S}_{b}$ e seus autovalores correspondentes não são nulos [Fukunaga 1990]. Para satisfazer essa condição foi utilizado uma extensão da técnica hamada MLDA - Maximum uncertainty LDA [Thomaz et al. 2006].

Assumindo que o espalhamento dos grupos de amostras se aproxima de uma distribuição Gaussiana [Tenorio and Thomaz 2011], o procedimento de navegar nas dimensões mais discriminantes pode ser descrito matematicamente por:

$$
\boldsymbol{y}=\overline{\boldsymbol{x}}+j \cdot \sigma_{i} \cdot \boldsymbol{P}_{p c a} \cdot \boldsymbol{w}_{m l d a},
$$

onde $\overline{\boldsymbol{x}}$ é a imagem média a ser navegada, $j \in\{-3,-2,-1,0,1,2,3\}, \sigma_{i}$ é o desvio padrão das amostras de cada grupo, $\boldsymbol{P}_{p c a}$ é a matriz de autovetores gerada pelo PCA e $\boldsymbol{w}_{m l d a}$ é o autovetor gerado pelo LDA (calculado através das amostras de imagens projetadas no espaço reduzido do PCA, computado intermediariamente antes do MLDA) [Thomaz et al. 2006].

Vale ressaltar que calcular o PCA como etapa intermediaria é necessário quando a dimensionalidade do espaço das características que representa uma imagem é muito maior do que o número total de imagens da amostra de treinamento $(n>>N)$. Quando o contrário é verdade $(N>n)$, essa etapa é desnecessária [Xavier et al. 2015].

\section{RESULTADOS}

As imagens originais de recém-nascidos do banco apresentam ausência de normalização, luminância, distâncias, ângulos e foco de captura diferentes, posicionamento, artefatos, oclusão da face, prejudicando análises diretas. Dadas essas condições iniciais algumas imagens foram retiradas do conjunto de treinamento e não foram utilizadas.

A partir do total de 360 imagens do banco, foram utilizadas ao todo 263 imagens para treinamento do modelo, sendo 121 imagens classificadas pelos profissionais de saúde como "com dor"e 142 imagens "sem dor". Essas imagens passaram pela etapa de préprocessamento e normalização do tamanho da imagem para 200x200 pixels.

Após segmentação das faces e redimensionamento é feito a marcação dos 68 pontos fiduciais, mapeando as principais regiões do rosto do recém-nascido para registro das imagens na etapa de normalização espacial. Exemplos de marcação desses pontos fiduciais sobre as faces das imagens pré-processadas estão ilustradas na Figura 3. 


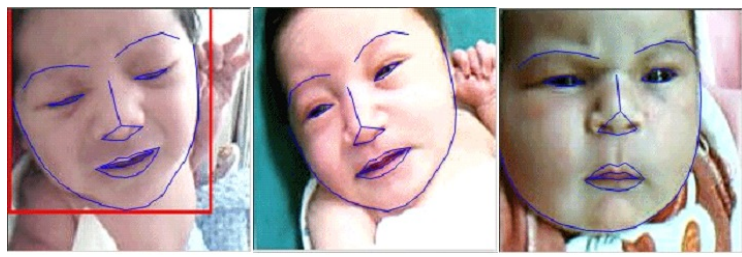

Figura 3. Três imagens de recém-nascidos com marcação dos pontos fiduciais.

Nesse ponto da metodologia proposta tem-se duas etapas. A primeira é referente a criação dos Atlas de Referência Inicial e Atlas de Recém-Nascidos, a segunda é o registro completo das imagens do banco em relação ao Atlas de Recém-Nascidos.

Nesse estudo o Atlas de Referência inicial foi criado a partir de algumas imagens alinhadas selecionadas aleatoriamente de cada classe, "Sem Dor"e "Com Dor".O fluxograma da metodologia aplicada e o Atlas de Referência Inicial de cada classe podem ser vistos na Figura 4.
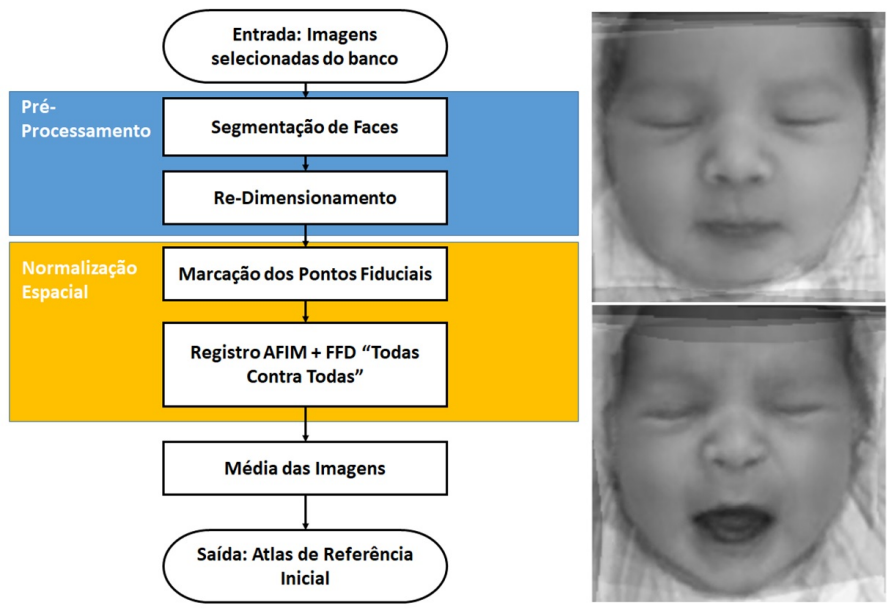

Figura 4. Fluxograma da metodologia utilizada para criação do Atlas de Referência Inicial, "Sem Dor"(imagem superior), "Com Dor"(imagem inferior).

A partir da criação dos Atlas de Referência Inicial foi possível construir o Atlas de Recém-Nascidos, para cada classe: "Sem Dor"e "Com Dor", a fim de obter uma imagem de referência mais genérica e com características específicas de cada classe. A metodologia utilizada e as imagens do atlas podem ser vistos no fluxograma da Figura 5.

Após a criação dos atlas e das imagens terem os pontos fiduciais marcados, as mesmas foram registradas primeiramente através de transformação rígida (AFIM) e depois através de transformação rígida e não-rígida (AFIM + FFD), em relação ao Atlas de Recém Nascidos referente a sua classe. A Figura 6 mostra um exemplo desta normalização.

Com as imagens normalizadas é possível extrair as características mais expressivas, utilizando PCA. A Figura 7 apresenta imagens criadas sinteticamente a partir da navegação nos hiperplanos extraídos do conjunto de imagens pelo PCA, descritos matematicamente na Equação (1). Cada linha de imagens representa a navegação de uma componente principal em ordem decrescente de variância, sendo a característica de maior 


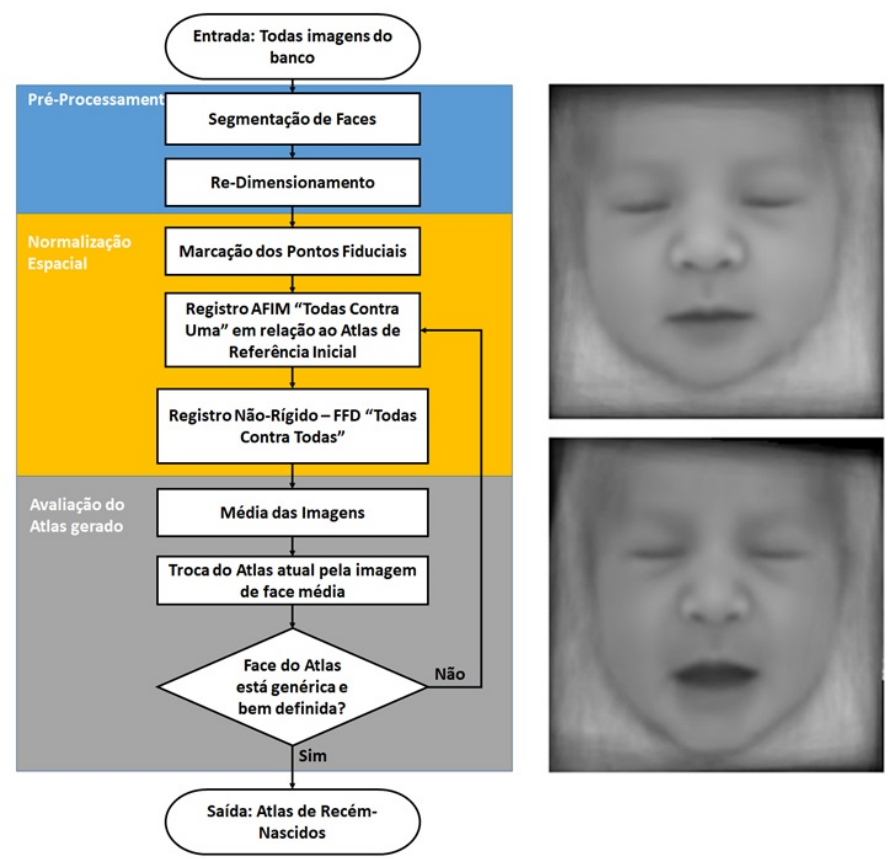

Figura 5. Fluxograma da metodologia utilizada para criação do Atlas de RecémNascidos, "Sem Dor"(imagem superior), "Com Dor"(imagem inferior).
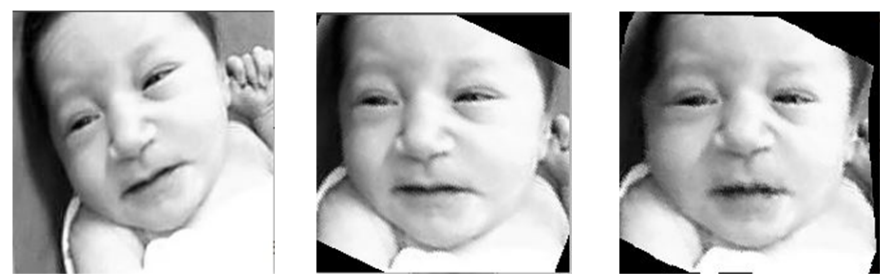

Figura 6. Exemplo de normalização Rígida e Não-Rígida.

variância correspondente à luminosidade. Contudo percebe-se que a segunda componente principal representa uma alteração de mímica facial de um recém-nascido sem dor para com dor, notando-se variações na boca, sulco nasolabial, olhos e sobrancelhas.

Os resultados apresentados pelo PCA mostram que a técnica não garante que todas características faciais estejam presentes em apenas uma componente principal e também não garante que as componentes de maior variância estejam relacionadas com as características a serem estudadas.

Portanto para encontrar um espectro contínuo onde existe uma única componente, sendo essa a mais discriminante entre as classes "Sem Dor"e "Com Dor", é aplicado a técnica MLDA. Na Figura 8 podem ser vistos os resultados das navegações geradas através da Equação (3), foram geradas 7 imagens sintéticas para essa navegação sendo possível observar alterações características nas mímicas faciais.

Diferente do que foi observado no resultado da navegação do PCA, com o MLDA é possível analisar as características mais discriminantes, eliminando dados e fatores de confusão que não são de interesse do estudo como a diferença de luminosidade entre as 


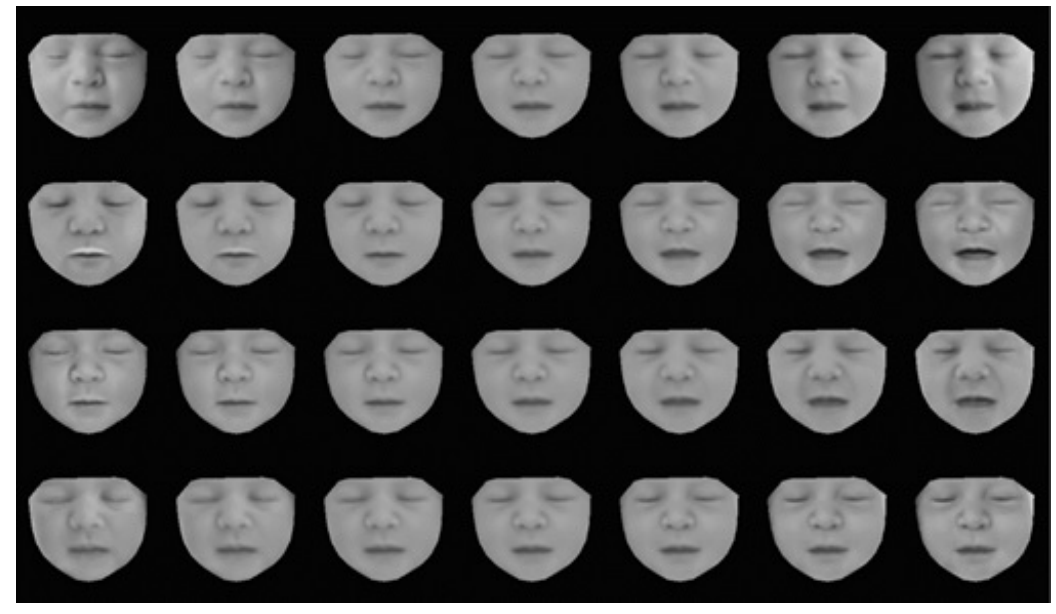

Figura 7. Imagens sintéticas resultantes da navegação nas componentes principais de 1 a 4 , descreve-se as características mais expressivas do conjunto de faces original.

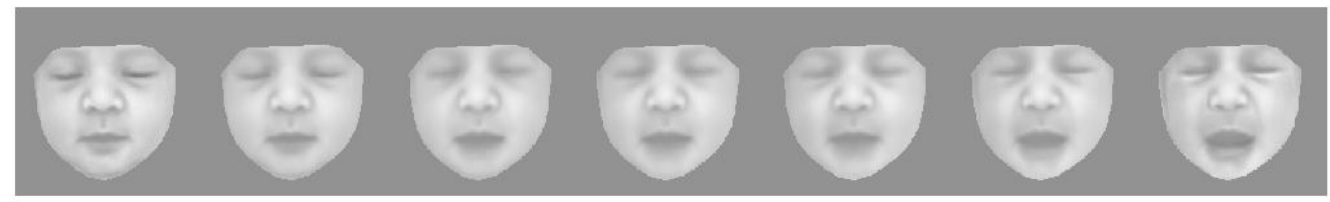

Figura 8. Características mais discriminantes, da esquerda para direita observase a evolução do estado sem dor para dor absoluta.

imagens.

Como método de avaliação dos resultados, foram relacionadas as classificações geradas através das características avaliadas utilizando a escala NFCS obtidas no relatório do estudo de [Heiderich et al. 2015] versus as classificações contínuas obtidas pelo MLDA na metodologia proposta, sendo possível verificar concordâncias e discordâncias entre as classificações. Para o conjunto de imagens utilizado no estudo, os resultados do MLDA em relação a classificação feita pelos profissionais foram idênticas para o conjunto de treinamento, mostrando que a modelagem da subjetividade humana na tarefa de classificação linear de dor em recém-nascidos foi exitosa. Já a acurácia, da relação entre o método proposto e a classificação utilizando o NFCS, foi de 72,77\%.

Através das análises das Figuras 9 e 10 é possível observar que as regiões de fronteira entre as classificações "Sem Dor"e "Com Dor"(NFCS = 2 e 4) apresentam maior discordância quando se é comparado a metodologia proposta versus a escala NFCS. Acredita-se que essa incerteza ou discordância de classificação tenha origem na subjetividade e dificuldade de identificar com precisão as características exatas das amostras próximas a fronteira entre "Sem Dor"e "Com Dor". Além disso existem outros fatores como, por exemplo, o estresse, que acredita-se possuir algumas características similares a classificação "Com Dor", gerando maior dificuldade na classificação binária "Sem Dor"e "Com Dor". 


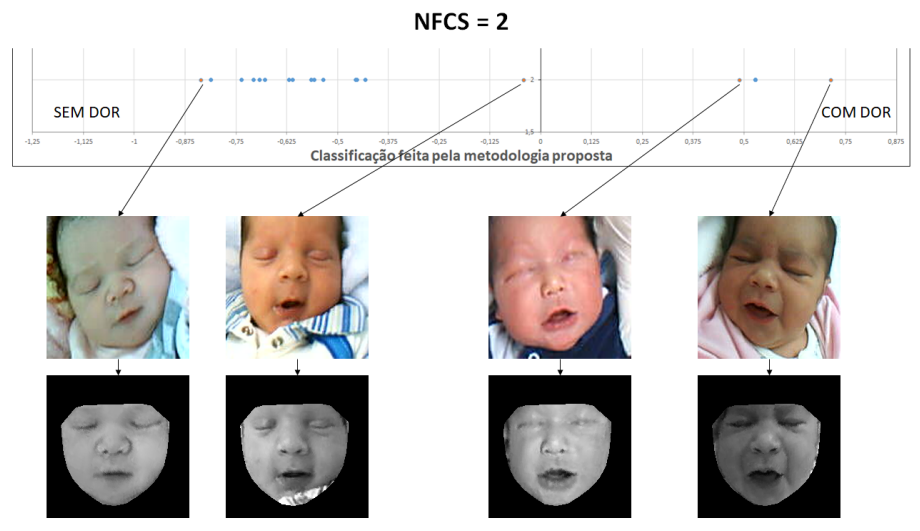

Figura 9. Relação entre a classificação das imagens pela escala NFCS com duas características positivas e MLDA.

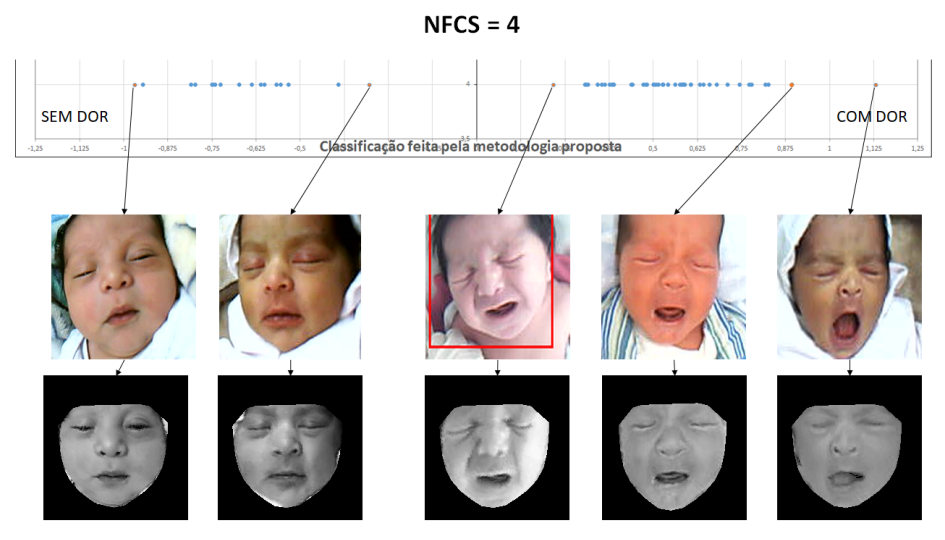

Figura 10. Relação entre a classificação das imagens pela escala NFCS com quatro características positivas e MLDA.

\section{CONCLUSÃO}

Na metodologia proposta foram utilizadas técnicas de pré-processamento e normalização de imagem, eliminando informações desnecessárias, segmentando faces e diminuindo desconformidades de escala, ângulo e morfologia facial. Essas técnicas foram essenciais para discriminar as imagens linearmente, permitindo classificações automáticas em espectros contínuos e possibilitando identificar o estado da dor do recém-nascido.

Os resultados apresentados no modelo possuem concordância em relação a classificação feita inicialmente por profissionais da área da saúde treinados para reconhecimento do fenômeno doloroso, permitindo uma melhor compreensão da subjetividade humana para essa tarefa de reconhecimento de dor. Já em relação a classificação da metodologia proposta versus a classificação dos mesmos profissionais utilizando NFCS, chegou-se a 72,77\% de acurácia. Acredita-se que essa discordância tenha origem em fatores de confusão não estudados aqui como, por exemplo, o estresse ou desconforto do recém-nascido. 


\section{Referências}

Amaral, V., Figaro-Garcia, C., Gattas, G. J. F., and Thomaz, C. E. (2009). Normalização espacial de imagens frontais de face em ambientes controlados e não-controlados. Periodico Cientifico Eletronico da FATEC Sao Caetano do Sul (FaSCi-Tech), 01:1-13. Acessado em Abril 4, 2017.

Anand, K. and Craig, K. (1996). New perspectives on the definition of pain. Pain, 67:3-6.

Aymar, C. and Coutinho., S. (2008). Fatores relacionados ao uso de analgesia sistêmica em neonatologia. Ver Bras Ter Intensiva, 20:405-415.

Chermont, A. G., Guinsburg, R., Balda, R. C., and Kopelman, B. I. (2003). O que os pediatras conhecem sobre avaliação e tratamento da dor no recém-nascido. Jornal Pediatr. (Rio J.), 79:265-272.

Dalal, N. and Triggs, B. (2005). Histograms of oriented gradients for human detection. In CVPR, pages 886-893.

Fisher, R. A. (1936). The use of multiple measurements in taxonomic problems. Annals of Eugenics, 7(7):179-188.

Fukunaga, K. (1990). Introduction to Statistical Pattern Recognition. Computer Science and Scientific Computing. Academic Press, Inc., 2nd edition.

Grunau, R. and Craig, K. (1987). Pain expression in neonates: facial action and cry. Pain, 28:395-410.

Grunau, R. E. (2013). Neonatal pain in very preterm infants: Long-term effects on brain,neurodevelopment and pain reactivity. Rambam Maimonides Med J., 25:4.

Guinsburg, R. (1999). Avaliação e tratamento da dor no recém-nascido. Jornal Pediatr. (Rio J.), 60:75-149.

Guinsburg, R. and Cuenca, M. (2010). A linguagem da dor no recém-nascido. Trends Genet., 1:1-5.

Heiderich, T. M., Leslie, A. T. F. S., and Guinsburg, R. (2015). Neonatal procedural pain can be assessed by computer software that has good sensitivity and specificity to detect facial movements. Acta Paediatrica, 104:63-69.

Kazemi, V. and Sullivan, J. (2014). One millisecond face alignment with an ensemble of regression trees. Proceedings of the 2014 IEEE Conference on Computer Vision and Pattern Recognition, pages 1867-1874.

Krechel, S. W. and Bildner, J. (1995). Cries: a new neonatal postoperative pain measurement score. initial testing of validity and reliability. Paediatr Anaesth, 5:53-61.

Lawrence, J., Alcock, D., McGrath, P., Kay, J., MacMurray, S., and Dulberg, C. (1993). The development of a tool to assess neonatal pain. Journal of Pain and Symptom Management, 6:59-66.

Luda, D., Nackley, A., Tchivileva, I., Shabalina, S., and Maixner., W. (2007). Genetic architecture of human pain perception. Trends Genet., 23:605-613.

Rueckert, D., Sonoda, L. I., Hayes, C., Hill, D. L. G., Leach, M. O., and Hawkes, D. J. (1999). Nonrigid registration using free-form deformations: Application to breast $\mathrm{mr}$ images. IEEE Transactions on Medical Imaging, 18:712-721. 
Sagonas, Tzimiropoulos, G., Zafeiriou, S., and Pantic, M. (2013). 300 faces in-the-wild challenge: The first facial landmark localization challenge. Proceedings of IEEE Int'l Conf. on Computer Vision (ICCV-W), 300 Faces in-the-Wild Challenge (300-W).

Stevens, B., Johnston, C., and Petryshen, P. (1996). Premature infant pain profile: development and initial validation. Research in Nursing Health, 12:13-22.

Tenorio, E. and Thomaz, C. (2011). Análise multilinear discriminante de formas frontais de imagens $2 \mathrm{~d}$ de face. Proceedings of the $X$ simpósio brasileiro de automação inteligente SBAI 2011, page 266-271.

Thomaz, C. E., Kitani, E., and Gillies, D. (2006). A maximum uncertainty lda-based approach for limited sample size problems - with application to face recognition. Journal of the Brazilian Computer Society, 12:7-18.

Xavier, I., Pereira, M., Giraldi, G., Gibson, S., Solomon, C., Rueckert, D., Gillies, D., and Thomaz., C. (2015). A photo-realistic generator of most expressive and discriminant changes in $2 \mathrm{~d}$ face images. In proceedings of the 6th International Conference on Emerging Security Technologies, EST-2015, 20:80-85.

Xavier, I. R. R., Giraldi, G. A., Gibson, S. J., Gattas, G. J. F., Rueckert, D., and Thomaz., C. E. (2016). Construction of a spatio-temporal face atlas: Experiments using down syndrome samples. 29th SIBGRAPI, Conference on Graphics, Patterns and Images, 29:1-4. 